\title{
197. Ästhetische Lidkorrektur unter Anwendung der myocutanen Lappenplastik
}

\author{
R. Stellmach
}

Klinik für Kiefer- und Plastische Gesichtschirurgie im Klinikum Steglitz der Freien Universität Berlin, Hindenburgdamm 30, D-1000 Berlin 45

\section{Cosmetic Blepharoplasty With the Muscle-Skin Flap Method}

Summary. Contrary to the standard method of stitch incision of the orbicularis muscle causing the danger of unequal lipectomy and bleeding that is difficult to control, a wide muscle incision from the nasal to the temporal end offers optimal access to the orbital fat prolaps. With the technique of Castanares a muscle-skin flap of lid skin and the outer part of the orbicularis muscle is developed, which after trimming the lid skin is pulled outwards slightly and resutured. In 30 consecutive cases this method has proved successful.

Key words: Cosmetic blepharoplasty.

Zusammenfassung. Im Gegensatz zum Standardverfahren der Stichincision des Orbicularismuskels mit der Gefahr ungleichmäBiger Fettentfernung und unzugänglicher Blutung entsteht bei einer breiten Muskelincision von medial nach lateral zur Korrektur von Tränensäcken volle Übersichtlichkeit und Zugänglichkeit zum Fettprolaps. Bei diesem Verfahren nach Castanares wird ein Myocutanlappen aus Lidhaut und dem peripheren Teil des Orbicularismuskels gebildet, der nach Kürzung der Lidhaut lateralisiert zurückgenäht wird. Das Vorgehen hat sich an 30 konsekutiven eigenen Fällen bewährt.

Schliisselwörter: Ästhetische Lidkorrektur - Blepharoplastik - Tränensäcke.

\section{Die Rekonstruktion des Unterlides bei tumorbedingten Defekten}

\section{J. Lentrodt}

Klinik für Kiefer- und Plastische Gesichtschirurgie der Universität Düsseldorf, Moorenstraße 5, D-4000 Düsseldorf

\section{Reconstruction of Lower Eye Lid After Tumour Surgery}

Summary. This is a report about the experience gained from the reconstruction of 274 lower eyelids after tumour surgery. Defects less than $12 \mathrm{~mm}$ allow primary wound closure. For greater defects several methods with flaps from the neighbourhood are available, whose indication is discussed. A specific procedure is presented in detail. The follow-ups have shown that it is possible to obtain a high percentage of good results both functionally as well as aesthetically, and this is illustrated with several examples.

Key words: Lid reconstruction.

Zusammenfassung. Es wird über Erfahrungen berichtet, die bei der Rekonstruktion von 274 Unterlidern nach tumoroperationsbedingter Defektsetzung gesammelt wurden. Bis zu einer Ausdehnung von $12 \mathrm{~mm}$ ist ein primärer Wundverschluß möglich. Für größere Defekte stehen zahlreiche Nahlappenmethoden zur Verfügung, deren Indikation erörtert wird. Ein eigenes Rekonstruktionsverfahren wird ausführlich geschildert. Die Nachuntersuchungen haben gezeigt, $\mathrm{da} B$ in einem hohen Prozentsatz sowohl funktionell als auch ästhetisch gute Ergebnisse erzielt werden können, was an zahlreichen Beispielen demonstriert wird.

Schlüsselwörter: Unterlidrekonstruktion. 